21st Particles and Nuclei International Conference (PANIC 2017)

International Journal of Modern Physics: Conference Series

Vol. 46 (2018) 1860002 (8 pages)

(C) The Author(s)

DOI: $10.1142 / \mathrm{S} 2010194518600029$

\title{
Double Beta Decay
}

\author{
Junpei Shirai \\ Research Center for Neutrino Science, Tohoku University, \\ Aoba-ku, Sendai-shi, 980-8578, Japan \\ shirai@awa.tohoku.ac.jp
}

Published 3 May 2018

\begin{abstract}
Double beta decay is a key process to reveal a fundamental property of neutrinos. If neutrinos are Majorana particles, that is they are equivalent to their antiparticles, neutrinoless double beta $(0 \nu \beta \beta)$ decay, $(A, Z) \rightarrow(A, Z+2)+2 e^{-}$, would occur. The process is beyond the standard model and would lead to a scenario which can explain the extremely small masses of neutrinos and provide a solution to the current matter dominance of the world. In this talk experimental efforts searching for $0 \nu \beta \beta$ decays are presented. Then, major $0 \nu \beta \beta$ experiments together with searches using ${ }^{136}$ Xe nuclei are described, followed by the current status of the KamLAND-Zen experiment.
\end{abstract}

Keywords: Mass of neutrinos; Majorana neutrinos; Neutrinoless double beta decays.

\section{Introduction}

It has been established that neutrinos have finite masses. Tremendous efforts have been made on the precise determination of the neutrino oscillation parameters including the challenges for finding the CP-phase and determination of the mass hierarchy of the neutrinos.

However, we do not know the absolute values of neutrino masses nor the reason of the size being so small compared to the charged leptons and quarks. Especially, it is not clear whether the neutrinos and the antineutrinos are equivalent (Majorana particles) or different (Dirac particles). Study of this problem is considered to be closely related to the origin of the neutrino masses; therefore, it is very important.

If neutrinos are Majorana particles, the mass term of the Lagrangian is generally given as

$$
-L_{m}=M_{\mathrm{D}}\left(\overline{\psi_{R}} \psi_{L}+\text { h.c. }\right)+\left(M_{L} / 2\right)\left(\overline{\left(\psi^{c}\right)_{R}} \psi_{L}+\text { h.c. }\right)+\left(M_{R} / 2\right)\left(\overline{\left(\psi^{c}\right)_{L}} \psi_{R}+\text { h.c. }\right),
$$

This is an Open Access article published by World Scientific Publishing Company. It is distributed under the terms of the Creative Commons Attribution 4.0 (CC-BY) License. Further distribution of this work is permitted, provided the original work is properly cited. 
where $M_{\mathrm{D}}$ is the Dirac mass, and $M_{L}$ and $M_{R}$ are the Majorana masses. By rearranging the fields two mass eigenstates are derived. Then, the physical masses $\left(M_{\nu}, M_{N}\right)$ are related by the well known see-saw mechanism, $M_{\nu}=M_{D}^{2} / M_{N}$. The relation can explain the extremely small masses of existing neutrinos by huge masses of the counter part which would have made crucial roles in the early stage of the Universe. In fact, today's matter dominance of the world could be explained by the CP violating decay of the super-heavy Majorana neutrinos. It would generate non-zero lepton number $(L)$, which is then converted to the baryon number $(B)$ through $\Delta(B-L)=0$. Therefore, Majorana nature of neutrinos is very important and should be checked experimentally.

\section{Double beta decay}

Nuclear double beta $(\beta \beta)$ decay provides the most sensitive and feasible way to study the Majorana nature of neutrinos. There are two kinds of $\beta \beta$ decays. One is the process $(2 \nu \beta \beta)$,

$$
(A, Z) \rightarrow(A, Z+2)+2 e^{-}+2 \bar{\nu}_{e}
$$

with emission of two neutrinos which is allowed by the SM. This occurs when the single beta decay is prohibited by the energy conservation and the spin states of the nuclei. It is the second order weak interaction, and the measured half-lives of more than ten nuclei are very long as typically $10^{19} \sim 10^{21} \mathrm{yr}$.

If neutrinos are Majorana particles, then the neutrino-less double beta $(0 \nu \beta \beta)$ decay would occur,

$$
(A, Z) \rightarrow(A, Z+2)+2 e^{-}
$$

This process violates the total lepton number and is beyond-the-SM process. Although not found yet, the decay mechanism is considered to be dominated by exchange of the light Majorana neutrinos. Then, the decay half-life $\left(T_{1 / 2}^{0 \nu}\right)$ is expressed as follows.

$$
\left(T_{1 / 2}^{0 \nu}\right)^{-1}=G^{0 \nu}(Q, Z)\left|M^{0 \nu}\right|^{2}\left\langle m_{\beta \beta}\right\rangle^{2},
$$

where the phase space factor $G^{0 \nu}(Q, Z)$ is reliably calculated; however, the nuclear matrix element (NME), $M^{0 \nu}$, has large theoretical uncertainty. The last factor, $\left\langle m_{\beta \beta}\right\rangle=\left|\Sigma U_{e i}^{2} m_{i}\right|$ is the effective Majorana neutrino mass. It contains all the information of the neutrinos including the absolute masses $\left(m_{i}\right)$, mixing matrix elements $\left(U_{e i}\right)$ which contain the neutrino mixing angles and the Dirac CP phase and the Majorana CP phases.

Figure 1 shows the lower limits on $T_{1 / 2}^{0 \nu}$ at $90 \%$ C.L. for the typical $\beta \beta$ nuclei obtained from recent experiments ${ }^{1,2,3,4,5,6,7}$ together with the measured values ${ }^{8}$ of $T_{1 / 2}^{2 \nu}$. The most stringent limit on $T_{1 / 2}^{0 \nu}(90 \%$ C.L.) is provided by KamLAND$\mathrm{Zen}^{6}$ as $>1.07 \times 10^{26}$ yr. Figure 2 shows the limits on $\left\langle m_{\beta \beta}\right\rangle$ as a function of the lightest neutrino mass. Depending on the mass hierarchy allowed regions are given 


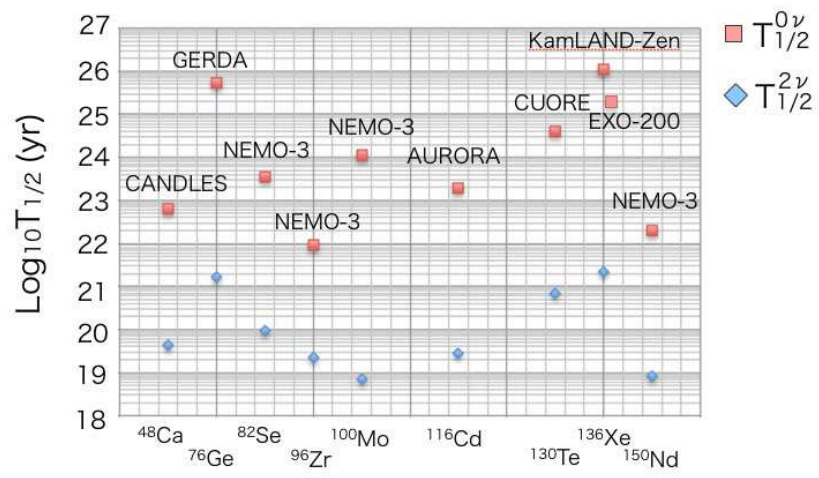

Fig. 1. Lower limits on the half lives of $0 \nu \beta \beta$ decays at $90 \%$ C.L. (red squares) and the measured $2 \nu \beta \beta$ decay half lives (blue diamonds) from recent $\beta \beta$ experiments for typical $\beta \beta$ nuclei.

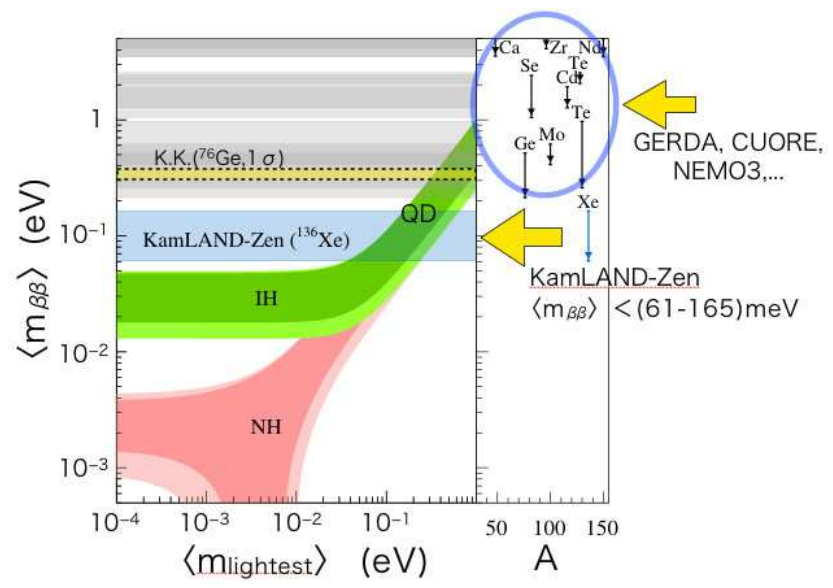

Fig. 2. Upper limits (90\%C.L.) on $\left\langle m_{\beta \beta}\right\rangle$ of recent $0 \nu \beta \beta$ search experiments and the allowed regions of the best fit (thick color) and $3 \sigma$ (thin color) for the normal mass hierarchy (NH), the inverted mass hierarchy (IH) and the quasi-degenerated (QD) regions provided by the neutrino oscillation experiments. The region of dashed horizontal lines (K.K.) indicates the positive claim for ${ }^{76} \mathrm{Ge}$ nucleus which is now refuted by recent high sensitivity experiments.

by the oscillation experiments. The stringent constraint by KamLAND-Zen of the limit $\left\langle m_{\beta \beta}\right\rangle<(61-165) \mathrm{meV}$ at $90 \%$ C.L. excludes most of the quasi degenerate (QD) region. The longstanding positive claim ${ }^{10}$ on the ${ }^{76} \mathrm{Ge}$ has been refuted by KamLAND-Zen and recent GERDA results.

\section{Experimental challenge to $0 \nu \beta \beta$ search}

The $0 \nu \beta \beta$ signal would appear as a peak of the summed electron energies at the decay energy $\left(Q_{\beta \beta}\right)$, where high-energy tail of the $2 \nu \beta \beta$ decays plus other backgrounds exist. The figure of merit of the $0 \nu \beta \beta$ sensitivity expressed for $T_{1 / 2}^{0 \nu}$ is 
shown as follows.

$$
T_{1 / 2}^{0 \nu} \propto \epsilon a \sqrt{\frac{M T}{b \Delta E}},
$$

where $M$ is the mass of the target material, $T$ is the experimental period, $b$ is the background index usually in the unit $\mathrm{keV}^{-1} \mathrm{~kg}^{-1} \mathrm{yr}^{-1}, \Delta E$ is the energy resolution or the region of interest (ROI), and $\epsilon$ and $a$ are the detection efficiency and the fraction (natural abundance or enrichment factor) of the isotope, respectively. It is clear that providing a large amount of the target nuclei, extreme rejection of backgrounds and good energy resolution are utmost important. Isotope selection by considering $a, Q_{\beta \beta}$ and $T_{1 / 2}^{2 \nu}$ is also important for selection of the nucleus.

Concept of the experiment is described as follows; preparation of a large amount of $\beta \beta$ isotope in deep underground, which is protected against internal and external radioactivity by thick shields of active and passive material with techniques to recognize the signal from backgrounds. Then excellent scalability is required for the system. As for the detection methods, calorimetric information by ionization, phonons and scintillation are obtained by using crystals, isotope-doped LS and TPCs (in gas and liquid phase). Additional information for PIDs by event topology together with informations of timing, position and pulse shape are employed for background rejection. Cutting edge technologies and new device are fully used to improve the energy resolution, detection efficiency and to get the scalability.

Next generation experiments are aiming at the half-life sensitivity of $10^{27} \mathrm{yr}$ using $\mathrm{O}(1)$ ton target. This would correspond to $\left\langle m_{\beta \beta}\right\rangle$ around $20 \mathrm{meV}$ covering the full inverted mass hierarchy $(\mathrm{IH})$ region. Many strategies are taken by current and planned high sensitivity experiments. Some of them are described in the next section.

\section{1. $0 \nu \beta \beta$ experiments with crystals}

GERDA $^{2}$ (Germanium Detector Array): Located in Gran Sasso underground facility $(3500$ m.w.e) they use 37 high purity germanium detectors of $35.6 \mathrm{~kg}$ in total enriched with ${ }^{76} \mathrm{Ge}\left(Q_{\beta \beta}=2039 \mathrm{keV}\right)$. The detectors are installed in the active shield of a $64 \mathrm{~m}^{3}$ liquid argon cryostat and surrounded by $590 \mathrm{~m}^{3}$ large water Cherenkov detector with a muon veto at the top. The Ge crystals are further protected against the background by fiber curtain. Excellent energy resolution is achieved around $2.8 \mathrm{keV}(\mathrm{FWHM})$ at $Q_{\beta \beta}$. The background rejection by the veto system and the PSD analysis are made. Recent results from the Phase $\mathrm{II}^{2}$ show that they realized the background-free search and provide the limit of $T_{1 / 2}^{0 \nu}>5.3 \times 10^{25} \mathrm{yr}(90 \%$ C.L.) corresponding to $\left\langle m_{\beta \beta}\right\rangle<(150-330) \mathrm{meV}$ (90\% C.L.).

The experiment will be scaled up to $200 \mathrm{~kg}$ crystal and finally planned to make new collaboration LEGEND with MAJORANA experiment aiming to the sensitivity for $\left\langle m_{\beta \beta}\right\rangle \sim(10-20) \mathrm{meV}$.

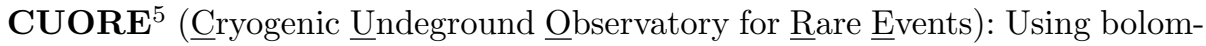
etry by ${ }^{n a t} \mathrm{TeO}_{2}$ crystals searching for $0 \nu \beta \beta$ decay of ${ }^{130} \mathrm{Te}$ (nat. ab. $=34 \%$, 
$\left.Q_{\beta \beta}=2527 \mathrm{keV}\right)$. The detector system have been developed from CUORICINO to CUORE0 and finally to CUORE made of $750 \mathrm{~kg}$ crystals and in a large cryostat which started operation for science run this year. Current limit on the half life at $90 \%$ C.L. by combining the previous results is $6.6 \times 10^{24} \mathrm{yr}$ corresponding to $\left\langle m_{\beta \beta}\right\rangle$ $<(210-590) \mathrm{meV}$. The challenging items are the long-term stable operation of the ton-scale bolometric detector and the validation of the background model in

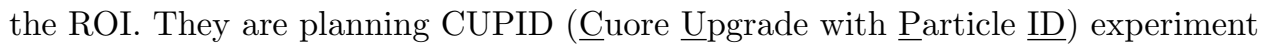
aiming to increase the source mass and reduce the backgrounds dramatically.

\section{2. $0 \nu \beta \beta$ experiments with ${ }^{136} X e$}

$0 \nu \beta \beta$ search using ${ }^{136} \mathrm{Xe}\left(Q_{\beta \beta}=2458 \mathrm{keV}\right.$, nat. ab.=8.9\%) benefits a lot from excellent chemical and physical properties of xenon as the noble gas. The enrichment and purification techniques of xenon are well established. The chemical stability provides high level of safety and easy handling. Moreover, emission of scintillation lights in the ionization in gas and liquid phase provide valuable information with TPC used in experiments like EXO-200, NEXT, PANDAX-III and many other planned experiments. Also, high solubility to the LS can provide another approach using a large volume LS detector like KamLAND-Zen experiment. These facts show that xenon provides excellent scalability. Another attractive point is that ${ }^{136} \mathrm{Xe}$ has a long $T_{1 / 2}^{2 \nu}$ of $2.1 \times 10^{21} \mathrm{yr}$, reducing $2 \nu \beta \beta$ background.

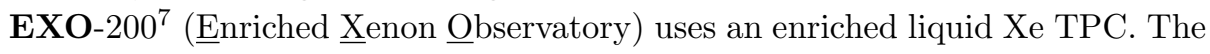
location is WIPP (Waste Isolation Pilot Plant) in New Mexico, in USA, with rock overburden of 1624 m.w.e. A good energy resolution of $1.2 \%$ at $Q_{\beta \beta}$ is obtained by combination of the scintillation and ionization signals. It also provides identification of $\alpha$ particles by the ratio of scintillation/ionization signals. $\beta / \gamma$ discrimination is made by topological information observed as single site vs multi site events. From the data taking in Phase-I and upgraded phase-II, they obtained lower limits on $T_{1 / 2}^{0 \nu}$ as $1.8 \times 10^{25} \mathrm{yr}(90 \%$ C.L. $)$. This corresponds to $\left\langle m_{\beta \beta}\right\rangle<(147-398) \mathrm{meV}(90 \%$ C.L.). They are planning the nEXO experiment with 5 ton liquid xenon at the SNOLAB aiming to $T_{1 / 2}^{0 \nu}$ sensitivity of $10^{28} \mathrm{yr}$.

NEXT $^{11}$ (Neutrino Experiment with a Xenon TPC) is an experiment of 10-bar Xe-gas TPC currently in R\&D stage in CANFRANC underground laboratory in Spain. The concept is that the scintillation light and electroluminescence in the multiplication of electrons are used for obtaining the topological information with a very good energy resolution. A proto-type detector of NEW(=NEXT-WHITE) of $50 \mathrm{~cm}$ drift length and $20 \mathrm{~cm}$ radius with $5-10 \mathrm{~kg}$ Xe is operated. The energy resolution of $5.5 \%$ for $41.5 \mathrm{keV}$ X-rays from ${ }^{83} \mathrm{Kr}$ is obtained which corresponds to $\sim 0.7 \%$ at $Q_{\beta \beta}$ (FWHM). They are planning NEXT-100 experiment using $100 \mathrm{~kg}$ enriched Xe aiming to the $T_{1 / 2}^{0 \nu}$ sensitivity of $5 \times 10^{25}$ yr corresponding to $\left\langle m_{\beta \beta}\right\rangle$ $\sim(90-180) \mathrm{meV}$.

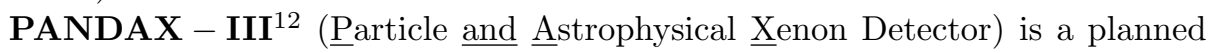
experiment of a 10-bar Xe-gas TPC using $200 \mathrm{~kg}$ enriched Xe. The TPC has the 
double sided drift region of $1 \mathrm{~m}$ drift length. The location is China Jinping Underground Laboratory (CJPL) in Sichuan in China, the world's deepest underground facility with a rock overburden of $6720 \mathrm{~m}$.w.e. where cosmic-ray muon flux is 0.2 $\mathrm{m}^{-2} \mathrm{day}^{-1}$. The prototype of the TPC is currently tested. The next step is to make a $200 \mathrm{~kg}$ Xe TPC module with the readout using Microbulk Micromegas. The final goal is a 1 ton Xe experiment by preparing the 5 TPC modules emersed in a water pool.

\subsection{KamLAND-Zen}

KamLAND-Zen (KamLAND Zero-Neutrino Double-Beta Decay) uses the KamLAND detector facility in Kamioka mine in Japan, where the rock overburden is 2700 m.w.e. As shown in Figure 3 a small balloon (mini-balloon) made of nylon film of $25 \mu \mathrm{m}$ thick is installed at the center of the detector and filled with Xe-dissolved LS using up to $380 \mathrm{~kg}$ of enriched Xe of $91 \%{ }^{136} \mathrm{Xe}$. It is surrounded by the 1000 ton ultrapure LS kept in a main balloon and viewed through buffer oil by PMTs at the inner surface of the stainless-steel spherical tank.

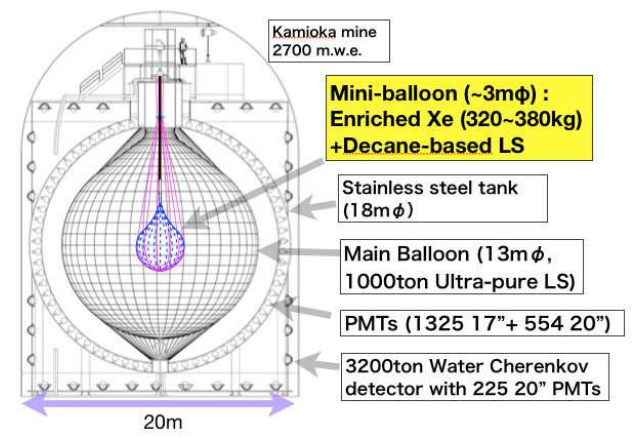

Fig. 3. Detector apparatus of KamLAND-Zen.

The system has excellent scalability and flexibility in carrying out the blank run and taking data using the outer LS in parallel. The amount of collected data since 2011 corresponds to ${ }^{136} \mathrm{Xe}$ exposure of $89.5 \mathrm{~kg}$ yr in phase I from October 2011 to June 2012 and $504 \mathrm{~kg}$ yr in phase II from December 2013 to October 2015. A dominant background from ${ }^{110 m} \mathrm{Ag}$ decays in phase I, which is considered from the fallout of the Fukushima reactor accident in 2011, was removed significantly by the Xe-LS purification carried out after the phase I. In phase II detailed studies on remaining background of ${ }^{214} \mathrm{Bi}$ on the balloon and rejection of muon-induced ${ }^{11} \mathrm{C}$ by the newly installed dead-time free electronics enabled a high quality analyses to provide the new limit on $T_{1 / 2}^{0 \nu}>1.07 \times 10^{26} \mathrm{yr}$ at $90 \%$ C.L. corresponding to $\left\langle m_{\beta \beta}\right\rangle>(61-165) \mathrm{meV}$ at $90 \%$ C.L. by combining the results of the phase I. 
KamLAND-Zen800 is a new phase using $750 \mathrm{~kg}$ of enriched Xe by replacing the balloon with a bigger and a cleaner one. Last summer the new balloon was deployed into the KamLAND detector. However, leaks were found after inflation with dummy LS and it was collected. However, the data collected in the period showed that the backgrounds of $\mathrm{U} / \mathrm{Th}$ series from the balloon films decreased to $\sim 1 / 3$ of the previous one showing the much better cleanliness of the balloon. Construction of a new balloon with improved fabrication methods was started. It will be deployed into KamLAND in a few months. We expect to $\left\langle m_{\beta \beta}\right\rangle$ sensitivity of $\sim$ (40-80) meV.

KamLAND2-Zen is a future upgrade version with much better energy resolution of $\sigma_{E} / E \sim 2 \%$ at $Q_{\beta \beta}$ to reduce the $2 \nu \beta \beta$ backgrounds aiming to fully cover the IH region. Many $\mathrm{R} \& \mathrm{Ds}$ are underway for the high quantum-efficiency PMTs with light collectors, high light-yield LAB-based LS, new LS purification methods to further remove radioactive elements of $\mathrm{U} / \mathrm{Th}$ series, and a new imaging sensor for $\beta / \gamma$ discrimination to reject backgrounds with much better efficiency.

\section{Outlook}

Current $0 \nu \beta \beta$ searches are improving the sensitivity to cover the $\mathrm{IH}$ mass region where some theoretical predictions suggest the $\left\langle m_{\beta \beta}\right\rangle$ value of several $10 \mathrm{meV}$ around the region. Constraint of the summed neutrino mass from the cosmological observation is consistent with the same $\left\langle m_{\beta \beta}\right\rangle$ values. Furthermore, coming neutrino oscillation experiments of accelerator, atmospheric, and reactor based ones will provide additional information on the mass hierarchy. We cannot give definite $\left\langle m_{\beta \beta}\right\rangle$ value; however, after improving the $0 \nu \beta \beta$ sensitivity there might be a big surprise !

\section{Summary}

- Majorana nature of neutrinos is a key to understand the fundamental questions of not only the particle physics but also origin of the Universe.

- $0 \nu \beta \beta$ decay is the beyond-the-SM process and the best feasible process to test the Majorana nature of neutrinos.

- Challenges to $0 \nu \beta \beta$ search are made worldwide using various nuclei with the mass of $\mathrm{O}(100) \mathrm{kg}$ to $\mathrm{O}(1)$ ton with cutting edge technologies to get the $\left\langle m_{\beta \beta}\right\rangle$ sensitivities in IH region.

- KamLAND-Zen with an unique strategy using Xe-doped LS will start a new $750 \mathrm{~kg}$ phase of enriched ${ }^{136} \mathrm{Xe}$ this year.

\section{Acknowledgments}

The author would like to thank the organizing committee for the excellent hospitality throughout the conference. 


\section{References}

1. CANDLES Collab., $\left({ }^{40} \mathrm{Ca}\right)$ Y.Takemoto, talk presented in this conference.

2. GERDA Collab., $\left({ }^{76} \mathrm{Ge}\right)$, M.Agostini et al. $)$, Nature 544, 47 (2017).

3. NEMO-3 Collab., $\left({ }^{82} \mathrm{Se}\right)$ A.S.Barabash et al., Phys. At. Nucl. 74, $312(2011) ;\left({ }^{96} \mathrm{Zr}\right)$ R.Arnold et al., Nucl. Phys. A847, 168 (2010); ( $\left.{ }^{100} \mathrm{Mo}\right)$ R.Arnold et al., Phys. Rev. D92, 072011 (2015); ( $\left.{ }^{150} \mathrm{Nd}\right)$ R.Arnold et al., Phys. Rev. D94, 072003 (2016).

4. AUROLA Collab., $\left({ }^{116} \mathrm{Cd}\right)$, F.A.Danevich et al., , arXiv:1601.05578.

5. CUORE Collab., $\left({ }^{130} \mathrm{Te}\right)$, C.Alduino et al., Eur. Phys. J. C77, 543 (2017).

6. KamLAND-Zen Collab., $\left({ }^{136} \mathrm{Xe}\right)$, A.Gando et al.,, Phys. Rev. Lett. 117, 082503 (2016).

7. EXO-200 Collab., $\left({ }^{130} \mathrm{Te}\right)$, J.B.Albert et al., arXiv:170\%.0870\%.

8. A.S.Barabash, Nucl. Phys. A935, 52 (2015).

9. KamLAND-Zen Collab., A.Gando et al.,, Phys. Rev. Lett. 110, 062502 (2013).

10. H.V.Klapdor-Kleingrothaus and I.V.Krivosheina, Mod. Phys. Lett. A21, 1547 (2006).

11. J.J.Gomez-Cadenas, talk given at the XLV Int. Meet. Fund. Phys., Granada, April, 2017.

12. Xun Chen et al., arXiv:1610.08883. 